\title{
Tumescent liposuction: A plastic surgeon's perspective
}

\author{
Walter Peters PhD MD FRCSC
}

$\mathrm{B}_{\mathrm{s}}^{\mathrm{r}}$ rown and colleagues (pages 69-74), from the United States Food and Drug Administration (FDA), have presented a comprehensive, well-written and somewhat sobering review of fatal and serious complications associated with liposuction. Suction lipectomy was initially performed in 1977 by Dr Yves-Gerard Illouz in Paris, France (1). A famous French movie actress asked him to remove a large lipoma, without leaving the usual long scar. He decided to try liposuction. Following the success of this patient, he soon used the procedure in many more cases. Initially, he used a dry technique. A few years later, he introduced his wet technique. The area to be suctioned was first injected with 100 to $300 \mathrm{~mL}$ of hypotonic saline containing hyaluronidase (1) to soften the fat and make it easier to aspirate. No adrenaline was used in this solution, so blood loss continued to be a problem. Aspirate analysis showed 70\% fat and 30\% blood $(1,2)$. The procedure was performed under general anesthesia. The removal of more than $1500 \mathrm{~mL}$ of aspirate was not recommended because of blood loss.

In October 1982, Illouz (1) presented his results from more than 3000 patients at the American Society of Plastic and Reconstructive Surgery Annual Meeting in Hawaii. Plastic surgeons from around the world were excited and began to perform suction lipectomy. By 1983, the adrenaline-containing wet technique emerged $(2,3)$. A volume of adrenaline-containing wetting solution (usually 100 to $300 \mathrm{~mL}$ ) was injected into the area that was to be aspirated. Subsequently, blood loss was reduced significantly and larger volumes of aspirate could be removed. However, periodic unpredictable bleeding was a significant impediment because of the relatively small volume of wetting solution.

In 1987, Dr Jeffrey Klein (4), a dermatologist, developed the technique that would revolutionize liposuction - the tumescent, or superwet, technique. This technique involved the injection of large volumes (usually two to three times the proposed aspirated volume) of wetting solution into subcutaneous tissues, until the skin was turgid. The solution contained highly-diluted lidocaine $(0.05 \%$ to $0.10 \%)$ and adrenaline $(1: 1,000,000)$. The tumescent technique provided three main advantages: it dramatically reduced blood loss, it provided local anesthesia and it facilitated fluid resuscitation. Aspirate blood content was reduced from $30 \%$ (with the Illouz techniques) to less than $1 \%(5,6)$. This reduction allowed much greater volumes of aspirate to be suctioned safely. The local anesthetic effect allowed the procedure to be performed without an anesthetist. It could be performed with or without intravenous sedation. Liposuction soon became the most commonly performed cosmetic surgery procedure in North America. 
Subsequently, larger and larger volumes of aspirate were removed routinely from patients. Procedures that were previously performed in hospitals (because of the requirement for an anesthetist) were now done in ambulatory surgery facilities and even in doctors' offices. By 1997, nearly half of the 250,000 liposuction procedures in North America were performed by office-based nonsurgeons, some with little more training than a weekend seminar (7). Monitoring of patients was not performed uniformly. Grazer and de Jong (8) showed that, among all physicians performing liposuction, most deaths $(47.7 \%)$ occur when the procedure is performed in a physician's office.

The illusion of technical simplicity led to the widespread perception that liposuction was atraumatic and risk-free. As the volumes of aspirate increased dramatically, greater numbers of serious complications, such as deep vein thrombosis, pulmonary embolus, fluid overload, pulmonary edema, lidocaine toxicity, necrotizing fasciitis and even death were reported (7,9). Brown et al (pages 69-74) indicate that there is a growing concern that serious or fatal outcomes are underreported.

Brown et al (pages 69-74) searched the Internet and found 39 deaths related to liposuction. They also analyzed five other studies with death rates ranging from 2.6/100,000 procedures to $100 / 100,000$ procedures. The largest study was a random survey from the American Society of Aesthetic Plastic Surgeons (8), which demonstrated 95 deaths in 496,000 procedures, for an incidence of $19.9 / 100,000$, or $1: 5224$. The highest death rate was $100 / 100,000(1: 1000)$ and was based on a study from a malpractice company insuring plastic surgeons in California. It should be emphasized that all of these studies contrast starkly with the $3: 100,000$ death rate for elective hernia surgery (8). Surprisingly, the reported liposuction fatality rates are very close to the $16.4 / 100,000$ fatality rate of motor vehicle accidents in the United States. Pulmonary thromboembolism appears to be the leading cause of liposuction-related death, followed by perforated viscus. However, it is known that deaths from anesthesia, sedation, lidocaine toxicity or respiratory insufficiency leave no anatomical findings and could well go undetected (8). Brown and colleagues (pages 69-74) stress the limitations of these studies. The studies are all retrospective, voluntary, under-reported and likely biased.

As the number of patients undergoing large-volume liposuction increases, the boundaries for what is recommended as safe are re-evaluated. Certain guidelines have been recommended by the American Society of Plastic Surgeons for tumescent liposuction (10). These guidelines provide the optimum level of safety for patients undergoing tumescent liposuction.

\section{PATIENT SELECTION}

Liposuction should not be considered as a treatment for general obesity. Obesity adds a substantial respiratory risk factor for respiration, sleep apnea, sedation and analgesics $(7,10)$. Liposuction should be used only on healthy patients
(American Society of Anesthesiologists Category 1) who are without significant underlying medical problems, and who are within $30 \%$ of their ideal body weights (7). The duration of all combined procedures should always be limited to less than $5 \mathrm{~h}$.

\section{MONITORING}

Careful monitoring is required for all patients undergoing intravenous sedation. This should involve a careful assessment and recording of cardiac rate, blood pressure, respiration rate, oxygen saturation, body temperature and level of consciousness (10). All injected fluids should be warmed to body temperature before injection. A warming blanket is necessary for all but minor procedures. If deep sedation is used, an anesthetist, who does not leave the patient's environment, needs to monitor the patient. Specific guidelines have also been developed for patient discharge (10).

\section{ASPIRATION VOLUME}

The tumescent technique has allowed much greater volumes of aspirate to be removed safely. A careful understanding of fluid management has increased the margin of safety. After aspiration, $60 \%$ to $70 \%$ of the injected fluid remains behind, to be absorbed from the tissues (7). Therefore, larger volume aspirations produce more fluid dislocations and have the potential for more serious complications. Although firm guidelines for fluid resuscitation have not been established, certain formulaic guidelines have proven to be useful when combined with good clinical judgment. In operations with low-to-medium volume aspirates (1000 to $4000 \mathrm{~mL}$ ), a 2:1 ratio of volume of subcutaneous fluid injection to volume of aspirate is recommended $(11,12)$, and intravenous volumes should not exceed $1000 \mathrm{~mL}$. Most procedures performed by plastic surgeons are in this group.

Patients undergoing large-volume aspirations (greater than $5000 \mathrm{~mL}$ ) require more extensive safety guidelines because larger volumes of infiltrate will be absorbed 12 to $24 \mathrm{~h}$ after surgery $(7,10)$. These procedures should be done only by a surgeon with considerable liposuction expertise. They require an anesthetist who is experienced in managing large-volume fluid shifts. The ratio of volume of subcutaneous fluid injection to volume of aspirate should approach 1:1 (12). No more than $500 \mathrm{~mL}$ of crystalloid should be given intravenously during the procedure. All these patients need to be catheterized and carefully monitored overnight as inpatients in a hospital setting or extended care facility (12).

Brown and colleagues (pages 69-74) raise particular levels of concern in their discussion of the lidocaine levels used with tumescent liposuction. The FDA-sanctioned maximum recommended adult dose of lidocaine (with adrenaline) for regional anesthesia is $7 \mathrm{mg} / \mathrm{kg}$. However, when injected for tumescent anesthesia, as a highly-diluted solution, at least five times that dose $(35 \mathrm{mg} / \mathrm{kg})$ has been used with impunity $(5,6)$. Even larger doses (55 to $80 \mathrm{mg} / \mathrm{kg}$ ) have been promoted as 'safe' in the sense that mean lido- 
caine blood levels remained below the putative $5 \mu \mathrm{g} / \mathrm{mL}$ cardiac threshold (13). Brown et al maintain that these levels of lidocaine (greater than $7 \mathrm{mg} / \mathrm{kg}$ ) are considered to be an 'off-label' use of lidocaine.

The main difference between lidocaine administered for regional nerve block and that for tumescent liposuction seems to reside in its dilution. Therefore, the FDA's $7 \mathrm{mg} / \mathrm{kg}$ maximum recommended adult dose must be heeded for undiluted, 'out-of-the-bottle' $0.5 \%$ to $2.0 \%$ lidocaine solution (with adrenaline). By contrast, a many-fold higher lidocaine dose ceiling of $35 \mathrm{mg} / \mathrm{kg}$ (perhaps more) applies specifically to extremely diluted $(0.02 \%$ to $0.05 \%)$ lidocaine solution (7). Currently, most plastic surgeons use a lidocaine concentration of $0.02 \%$ ( $20 \mathrm{~mL}$ of $1 \%$ lidocaine in $1 \mathrm{~L}$ of Ringer's lactate solution). The working hypothesis for this lidocaine-dosing paradox is that the injected tissues have an intrinsic binding capacity for the dilute lidocaine. This slows its release from tissue and increases its safety $(4,6)$. A similar sequestration mechanism has been suggested for the high levels of adrenaline, because tachycardia, arrhythmia and hypertension are generally not observed clinically.

The incidence of serious complications from liposuction has certainly increased dramatically over the years. However, when the procedure is performed by fellowshiptrained surgeons, in accredited surgical facilities, and with close attention to the established safety guidelines (10), then liposuction is usually an inherently safe procedure.

The main goals of the review by Brown et al (pages 69.

74) are to encourage physicians and health care providers

\section{REFERENCES}

1. Illouz YG. History and current concepts of lipoplasty. Clin Plast Surg 1996;23:721-30.

2. Hetter GP. Blood and fluid replacement for lipoplasty procedures. Clin Plast Surg 1989;16:245-8.

3. Fodor PB. Defining wetting solutions in lipoplasty. Plast Reconstr Surg 1999;103:519-25.

4. Klein JA. The tumescent technique for liposuction surgery. Am J Cosmetic Surg 1987;4:263-70.

5. Klein JA. Tumescent Technique: Tumescent Anesthesia and MicroCannular Liposuction. St Louis: Mosby, 2000.

6. Rohrich RJ, Beran SJ, Fodor PB. The role of subcutaneous infiltrate in suction-assisted lipoplasty: A review. Plast Reconstr Surg 1997;99:514-9.

7. de Jong RH, Grazer JM. Perioperative management of cosmetic liposuction. Plast Reconstr Surg 2001;107:1039-44.

8. Grazer FM, de Jong RH. Fatal outcomes from liposuction: Census to report adverse reactions from liposuction, to promote more research on the complications of liposuction, and to alert physicians to the problems associated with liposuction, so that they may better inform their patients. As plastic surgeons, we certainly strongly support these goals. There are many areas that require further study. Brown et al (pages 69-74) stress the need for prospective studies that examine potential risk factors and the risk/benefit profile for liposuction patients.

The potential role of lidocaine cardiotoxicity is widely underappreciated. For instance, although lidocaine megadosing has been heralded as 'safe', that inference is based only on limited, serial blood level measurements (7). There have been no vigorous pharmokinetic studies of lidocaine disposition or metabolism. There has also been no definitive appraisal of the cardiovascular response to sedative or anesthetic agents superimposed on a persistent background of elevated levels of blood lidocaine. Little is known about the deportation of fat globules in the bloodstream. It seems logical that some fat globules mobilized by cannula trauma could deport into the general circulation as blood vessels are disrupted. This could be more common when fat is emulsified by ultrasonic extraction (7). Since the review of Brown et al (pages 69-74) was submitted, one ultrasonic unit has been approved by the FDA - the Mentor Contour Genesis System (Mentor Corporation, USA), which was approved in October 2001. Further investigation in these areas may lead to a greater understanding of the pathophysiology and safety of liposuction.

survey of cosmetic surgeons. Plast Reconstr Surg 2000;105:436-46.

9. Commons GW, Halperin B, Chang CC. Large-volume liposuction: A review of 631 consecutive cases over 12 years. Plast Reconstr Surg 2001;108:1753-67.

10. Iverson RE. Sedation and analgesia in ambulatory settings. American Society of Plastic and Reconstructive Surgeons. Task Force on Sedation and Analgesia in Ambulatory Settings. Plast Reconstr Surg 1999;104:1559-64.

11. Pitman GH, Aker JS, Tripp ZD. Tumescent liposuction. A surgeon's perspective. Clin Plast Surg 1996;23:633-41.

12. Pitman GH. Large-volume liposuction: A review of 631 consecutive cases over 12 years - Invited Discussion. Plast Reconstr Surg 2001;108:1764-5.

13. Ostad A, Kageyama N, Moy RL. Tumescent anesthesia with a lidocaine dose of $55 \mathrm{mg} / \mathrm{kg}$ is safe for liposuction. Dermatol Surg 1996;22:921-5. 